INPLASY

PROTOCOL

To cite: Wang et al. Effect of Ganduqing on Common Cold: A Protocol for Systematic Review and Meta-analysis Based on Existing Evidence. Inplasy protocol 202060073. doi:

10.37766/inplasy2020.6.0073

Received: 20 June 2020

Published: 20 June 2020

Corresponding author:

Fei Wang

wangfei2020007@163.com

Author Affiliation:

Hospital of Chengdu

University of TCM

Support: MIPFSATOSP (Grant No. 2019055)

Review Stage at time of this submission: Data analysis.

Conflicts of interest:

This work has no conflicts of interest.

\section{Effect of Ganduqing on Common Cold: A Protocol for Systematic Review and Meta-analysis Based on Existing Evidence}

Wang, X1; Wu, Y2; Huang, D³; Pei, C4; Wang, Z5; Wang, M6.

Review question / Objective: This review aims to systematically evaluate the benefits and harms of Ganduqing for common cold patients reported in randomized clinical trials (RCTs).

Condition being studied: The common cold is a common upper respiratory tract disease, usually caused by rhinovirus infection, which is self-limiting, and its main symptoms include sore throat, rhinitis, runny nose, fever, etc. Although the common cold is self-limiting, when the symptoms are severe, the productivity of the patient will be reduced. Ganduqing is an herbal formula composed of (Astragalus, Shegan) and several randomized controlled trials have been carried out, so it is necessary to conduct a systematic review to evaluate the effectiveness and safety of Ganduqing in the management of common colds.

INPLASY registration number: This protocol was registered with the International Platform of Registered Systematic Review and Meta-Analysis Protocols (INPLASY) on 20 June 2020 and was last updated on 20 June 2020 (registration number INPLASY202060073).

\section{INTRODUCTION}

Review question / Objective: This review aims to systematically evaluate the benefits and harms of Ganduqing for common cold patients reported in randomized clinical trials (RCTs).
Condition being studied: The common cold is a common upper respiratory tract disease, usually caused by rhinovirus infection, which is self-limiting, and its main symptoms include sore throat, rhinitis, runny nose, fever, etc. Although the common cold is self-limiting, when the symptoms are severe, the productivity of 
the patient will be reduced. Ganduqing is an herbal formula composed of (Astragalus, Shegan) and several randomized controlled trials have been carried out, so it is necessary to conduct a systematic review to evaluate the effectiveness and safety of Ganduqing in the management of common colds.

\section{METHODS}

Participant or population: The patients of common cold (using 2012 edition of "Expert Consensus for the Standard Diagnosis and Treatment of Common Colds"). These types of patients will not be included: Influenza patients; patients with the total number of white blood cells in routine blood examination $>12.0 \times 109 / L$, and the percentage of neutrophils $>80 \%$; patients severe heart disease, liver and kidney dysfunction, mental illness, or a relevant drug allergic history and patients during pregnancy or lactation.

Intervention: Ganduqing or modified Ganduqing.

Comparator: Lianhua Qingwen Capsule and Compound Paracetamol Capsules.

Study designs to be included: Only randomized controlled trial will be included.

Eligibility criteria: Only RCTs (except QuasiRCTs and cluster RCTs) will be included. Animal mechanism studies and nonrandomised clinical trials will be excluded. Article that substantially overlaps with another published article in print or electronic media will be excluded. Duplicate publications produced by a single experiment and published as separate papers with different criteria for measuring results, priority will be given to original publications and other publications will be excluded. The language and time of publication will not be restricted.

Information sources: This review will include grey literature sourced from CCPD (China Conference Paper Database), manual searching. Electronic database includes PubMed, Embase, Cochrane
Library, Web of Science, CNKI, WanFang, VIP and CBM will also be searched.

Main outcome(s): The main outcomes include the improvement of cough, sore throat, runny nose, fever, and nasal congestion and after taking the drug, the blood routine examination of neutrophil percentage and lymphocyte percentage changes in patients with common cold.

Additional outcome(s): After taking the medicine, the effective patients relapsed from the common cold.

Quality assessment / Risk of bias analysis: All the included studies will be evaluated based on the guidelines of Cochrane Handbook for Systematic Reviews of Interventions. The quality of each trial will be categorized into 'low', 'unclear', or 'high' risk of bias according to the following items: adequacy of generation of the allocation sequence, allocation concealment, blinding of participants and personal, blinding of outcome assessors, incomplete outcome data, selected reporting the results and other sources of bias (such as comparable baseline characteristic, inclusion and exclusion criteria.

Strategy of data synthesis: We used Revman 5.3 software provided by the Cochrane collaboration and Stata 14 software to analyze the data. Binary outcomes will be summarized using risk ratio (RR) with $95 \%$ confidence interval $(\mathrm{Cl})$ for relative effect. Continuous outcomes will be summarized by using weighted mean difference (WMD) with $95 \% \mathrm{Cl}$. We will use random-effect model (REM) for meta-analysis in this review according to research recommendations. Statistical heterogeneity will be assessed by $X^{2}$ and 12 statistical tests. Where $p$ value $\geq 0.1$ and 12 $\leq 50 \%$, there is no obvious statistical heterogeneity among the studies. On the contrary, where $p$ value $50 \%$ indicates a considerable heterogeneity. Meta-analysis will be performed when the statistical heterogeneity is acceptable ( $p$ value $\geq 0.1$ and $12 \leq 50 \%$ ), otherwise, subgroup analysis will be applied to explore the 
influence of potential factors on the outcome measures.

Subgroup analysis: We will conduct Subgroup analyses by different age, gender, course of treatment, and different type of Ganduqing (intervention forms, pharmaceutical dosage form, dosage, etc). If a meta-analysis cannot be performed, we will conduct descriptive analysis instead.

Sensibility analysis: We will conduct sensitivity analyses by omitting studies one by one to probe the impact of an individual study.

Language: Chinese.

Country(ies) involved: China.

Keywords: Ganduqing; common cold; Systematic review.

Contributions of each author:

Author 1 - Xiaomin Wang.

Author 2 - Yongcan Wu.

Author 3 - Demei Huang.

Author 4 - Caixia Pei.

Author 5 - Zhenxing Wang.

Author 6 - Mingjie Wang. 